\title{
The selective processing of briefly presented affective pictures: An ERP analysis
}

\author{
HARALD T. SCHUPP, ${ }^{\mathrm{a}, \mathrm{b}}$ MARKUS JUNGHÖFER, ${ }^{\mathrm{b}}$ ALMUT I. WEIKE, ${ }^{\mathrm{a}}$ AND ALFONS O. \\ HAMM $^{\mathrm{a}}$ \\ ${ }^{\mathrm{a}}$ Department of Psychology, University of Greifswald, Greifswald, Germany \\ ${ }^{\mathrm{b}}$ Department of Psychology, University of Konstanz, Konstanz, Germany
}

\begin{abstract}
Recent event-related potential (ERP) studies revealed the selective processing of affective pictures. The present study explored whether the same phenomenon can be observed when pictures are presented only briefly. Toward this end, pleasant, neutral, and unpleasant pictures from the International Affective Pictures Series were presented for $120 \mathrm{~ms}$ while event related potentials were measured by dense sensor arrays. As observed for longer picture presentations, brief affective pictures were selectively processed. Specifically, pleasant and unpleasant pictures were associated with an early endogenous negative shift over temporo-occipital sensors compared to neutral images. In addition, affective pictures elicited enlarged late positive potentials over centro-parietal sensor sites relative to neutral images. These data suggest that a quick glimpse of emotionally relevant stimuli appears sufficient to tune the brain for selective perceptual processing.
\end{abstract}

Descriptors: Emotion, Attention, Motivation, Perception, Event-related potential, Affect

Affective pictures are effective cues to engage emotional response output systems (Lang, Bradley, \& Cuthbert, 1997). Accordingly, affective pictures consistently modulate autonomic measures (electrodermal responses and heart rate), facial EMG responses (corrugator and zygomaticus), and the startle blink reflex (cf. Bradley, 2000; Hamm, Schupp, \& Weike, 2003). More recent studies determined that briefly presented affective pictures also engage these autonomic, facial, and reflex responses, in many ways similar to more sustained presentations (Codispoti, Bradley, \& Lang, 2001; Globisch, Hamm, Esteves, \& Öhman, 1999). Focusing on the perceptual/evaluative stage of picture processing, recent event-related brain potential (ERP) studies explored the cortical processing of affective stimuli (e.g., Cuthbert, Schupp, Bradley, Birbaumer, \& Lang, 2000; Schupp, Junghöfer, Weike, \& Hamm, 2003a). In general, the results were consistent with the hypothesis that affective pictures draw attentional resources (Lang et al., 1997). Continuing this line of research, the present study explored whether briefly presented affective pictures are also selectively processed.

A number of recent ERP studies explored emotional picture processing by presenting pleasant, neutral, and unpleasant

This research was supported by grants from the German research foundation Schu1074/7-1 to Harald Schupp and Ha1593/10-2 to Alfons Hamm. We thank Jessica Stockburger and Johannes Bahlmann for their assistance in data collection.

Address reprint requests to: Harald T. Schupp, Institute of Psychology, Department of Experimental Psychology, University of Konstanz, Mailbox D36, 78457 Konstanz, Germany. E-mail: Harald.Schupp@, uni-konstanz.de. pictures from the International Affective Picture Series (IAPS; Cuthbert et al., 2000; Keil et al., 2002; Schupp et al., 2000, 2003a; Schupp, Cuthbert, et al., in press). For instance, Cuthbert et al. (2000) observed that emotionally arousing (pleasant and unpleasant) pictures elicited enlarged late positive potentials (LPPs) compared to neutral images, which developed around 350-400 ms and lasted for several hundred milliseconds. Moreover, this phenomenon was related to the intensity of the emotional stimulation. That is, unpleasant and pleasant pictures of high emotional arousal elicited larger LPP amplitudes than less arousing pictures of the same valence (Cuthbert et al., 2000). Continuing this line of research, Schupp et al. (2000) replicated these findings by presenting affective pictures in a random sequence using the timing parameters of the modified oddball paradigm (cf. Cacioppo, Crites, Berntson, \& Coles, 1993). In this paradigm, pictures are presented in sequences of six exemplars with individual pictures shown for $1.5 \mathrm{~s}$ at a relatively rapid rate (intertrial intervals [ITI] vary between 1.5 and $3 \mathrm{~s}$ ). Again, enlarged LPP amplitudes were observed for pleasant and unpleasant compared to neutral pictures, and this affective modulation was particularly pronounced for high-arousing affective pictures. In cognitive research, it was found that positive late potentials reflect further processing related to the perceptual demands of the task after the initial stimulus identification is completed (e.g., Ritter \& Ruchkin, 1992). According to these findings, it was suggested that the intrinsic relevance of emotional stimuli initiates the continued perceptual analysis of these stimuli (cf. Cuthbert et al., 2000). This effect has been theoretically linked to the concept of motivated attention, assuming that 
motivationally significant stimuli are selectively processed because they draw attentional resources (Lang et al., 1997).

However, recent dense sensor ERP studies also provided evidence for an early selective processing of affective cues. Previous ERP studies used only a sparse number of sensors, and thus, were limited in exploring the spatial topography and temporal course of selective affective processing, and in particular, selective stimulus processing at an earlier level of stimulus processing. Therefore, Schupp et al. (2003a) recently investigated electrocortical activity during processing of emotional pictures using multichannel ERP recordings (129 sensors). This methodology does not only provide an improved spatial resolution but also permits a reference-independent characterization of the brain potentials (cf. Junghöfer, Elbert, Leiderer, Berg, \& Rockstroh, 1997; Tucker, Liotti, Potts, Russell, \& Posner, 1994). A random series of pleasant, neutral, and unpleasant pictures was presented. Again, pictures were shown for $1.5 \mathrm{~s}$ in streams of six images. Replicating previous findings, augmented LPP amplitudes were observed for affective relative to neutral pictures. Additionally, pictures were found to modulate ERPs at an earlier stage of stimulus processing. Pleasant and unpleasant pictures elicited an endogenous negative shift over temporo-occipital sites that started around $150 \mathrm{~ms}$ after picture onset and reached its peak around $300 \mathrm{~ms}$. Furthermore, this early posterior negativity (EPN) increased with the arousal level of the emotional pictures. That is, higharousing picture contents of erotic scenes and mutilations elicited a more pronounced posterior negativity compared to less arousing categories of the same valence. Taken together, the selective processing of emotional pictures is reflected by increased EPN and LPP amplitudes.

The present study explored the selective processing of briefly presented affective pictures at the level of the perceptual/ evaluative stage of information processing (cf. Cacioppo, Gardner, \& Berntson, 1999). Exploring this issue follows the lead of two recent studies determining whether brief picture presentations of emotional pictures are sufficient to engage defensive and appetitive motivation as indicated by emotional response output measures (Codispoti et al., 2001; Globisch et al., 1999). Studying affective responses to brief presentations of visual cues (500 ms), Codispoti et al. (2001) observed greater skin conductance responses for emotional pictures and increased corrugator responses for unpleasant pictures, similar to more sustained presentations. In addition, startle reflexes were larger for unpleasant compared to pleasant pictures. However, in contrast to longer presentations, startle reflexes to unpleasant pictures were not potentiated relative to neutral images, indicating less defensive activation prompted by brief presentations. No such difference between brief $(150 \mathrm{~ms})$ and longer presentations $(6,000 \mathrm{~ms})$ were observed when snake/spider phobic subjects processed fear-relevant pictures (Globisch et al., 1999). In this study, both brief and long picture presentations induced potentiated startle reflexes for fear-relevant relative to neutral images in animal-fearful participants. Moreover, startle potentiation was already observed $300 \mathrm{~ms}$ after picture onset, suggesting a fast appearance of defensive motivation with more intense unpleasant stimuli. Taken together, these studies provide evidence that brief presentations of affective pictures do engage appetitive and defensive motivation. In particular, interpretation of these studies stressed that "affective pictures draw more heavily on attentional resources at encoding than do neutral pictures" (Codispoti et al., 2001, p. 477).
Accordingly, the present study examined whether selective processing of emotional pictures can be observed when pictures are presented briefly. Towards this end, pleasant, neutral, and unpleasant pictures were randomly presented in sequences of six exemplars while ERPs were measured with a dense sensor electrode array. In contrast to previous studies (Schupp et al., 2000, 2003a), pictures were presented for only $120 \mathrm{~ms}$ (instead of $1.5 \mathrm{~s}$ as in previous studies). The brief presentation time used in this study allowed for a single fixation of the pictures only (cf. Christianson, Loftus, Hoffman, \& Loftus, 1991). Selective processing at the perceptual stage of information processing was predicted to be associated with increased EPN amplitudes for pleasant and unpleasant pictures compared to neutral contents. Similarly, increased LPP amplitudes to pleasant and unpleasant relative to neutral images would indicate late selective processing of briefly presented affective pictures. Further analyses determined if these early and late selective modulations vary as a function of emotional intensity, that is, whether they were particularly pronounced for pictures of erotica and mutilations.

\section{Methods \\ Participants}

Twenty volunteers from the University of Greifswald introductory psychology course participated as part of a class requirement. The data from four participants were excluded from analysis because of excessive blink artifacts $(n=2)$ or incomplete data $(n=2)$, leaving 16 participants (14 women) for the final sample. Participants were between the ages of 19 and 27 years $(M=21.2$ years).

\section{Stimulus Materials}

Participants viewed 60 pictures from the International Affective Picture System (IAPS; Lang, Bradley, \& Cuthbert, 1999), a collection of standardized photographic materials. Pleasant picture contents included high-arousing images displaying heterosexual couples in erotic postures $(n=10)$ and low-arousing images of babies and family scenes $(n=10)$. Neutral images were either showing household objects $(n=10)$ or pictures of people posing neutral expressions $(n=10)$. Unpleasant images included images of mutilations $(n=10)$ and threat (animal and human threats; $n=10$ ). These images had simple figure/ground distinctions and were similar in content to the pictures used in previous functional magnetic resonance imaging (fMRI) and EEG studies. In data analysis, neutral household items were considered separate to avoid confounds with specific stimulus features of this particular picture category (e.g., reduced perceptual complexity). Therefore, only colored pictures of humans in different situations entered the analyses reported here. The three categories differed significantly from each other in IAPS normative valence ratings $(M=7.3,4.8$, and 2.3 for pleasant, neutral, and unpleasant contents). Mean arousal levels for both emotional categories were significantly higher than for neutral contents $(M=5.4,3.0$, and 6.8 for pleasant, neutral, and unpleasant contents, respectively).

\section{Procedure}

Participants were seated in a recliner in a dimly lit, soundattenuated room. After filling out the informed consent form, the geodesic sensor net was attached, and task instructions were given. 
The visual stimuli were projected on a white screen located approximately $1.3 \mathrm{~m}$ from the participant's eyes ( $\approx 24^{\circ}$ of visual angle) using a Kodak Ektapro 5000 slide projector. Pictures were displayed for $120 \mathrm{~ms}$ and presented serially in sequences of six pictures (cf. Cacioppo et al., 1993). To reduce the effects of stimulus offset, the brightness of the pictures was smoothly reduced in the time window from 80 to $120 \mathrm{~ms}$. A warning dot preceded picture onset to ensure that participants were fixating on the center of the screen. As in previous studies (Schupp et al., 2000, 2003a), an evaluation task was used to ensure that the participant was attending to the pictures. Thus, the participant was asked to categorize each picture either as pleasant, neutral, or unpleasant using a three-way response button. To avoid contamination of the ERP in the LPP time window with movement artifacts, participants were asked to withhold the rating until a soft tone presented $1.5 \mathrm{~s}$ after picture onset signaled the beginning of the subjective rating period. The participant initiated each sequence of six pictures by a button press, and a total of 40 such sequences were presented. Thus, each picture was viewed four times in the total of 240 picture presentations. The order of pleasant, neutral, and unpleasant pictures was perceptually random within a sequence. According to normative group ratings (Lang et al., 1999), sequences of at most two pictures of the same valence category based on normative ratings were allowed. All 60 different exemplars were presented before the picture series was presented again (in a different order). Each particpant received his or her own order of picture presentation. One sequence of six pictures served as a practice trial.

\section{Data Collection and Analysis}

Electrophysiological data were collected from the scalp using a 129-channel system (Electrical Geodesics, Inc., Eugene, OR). Scalp impedance for each sensor was kept below $30 \mathrm{k} \Omega$. Due to the high input impedance of the EGI amplifier, scalp impedances below $50 \mathrm{k} \Omega$ are recommended to ensure an optimal signal-tonoise ratio for this amplifier system. The EEG was recorded continuously with a sampling rate of $250 \mathrm{~Hz}$, the vertex sensor as reference electrode, and on-line bandpass filtered from 0.01 to $100 \mathrm{~Hz}$. Continuous EEG data were low-pass filtered at $35 \mathrm{~Hz}$ using a zero-phase forward and reverse digital filter before stimulus synchronized epochs were extracted from $124 \mathrm{~ms}$ before until $776 \mathrm{~ms}$ after picture onset. Single trial epochs were corrected for vertical and horizontal eye movements using a correlative eye movement algorithm (Miller, Gratton, \& Yee, 1988). The criteria that all sensors are required to be artifact free in a given trial would result in an unacceptably low number of trials using dense sensor ERP recordings. To deal with this problem, data editing and artifact rejection were based on the method for statistical control of artifacts (Junghöfer, Elbert, Tucker, \& Rockstroh, 2000). This procedure (1) detects individual channel artifacts using the recording reference, (2) detects global artifacts using the average reference, (3) replaces artifact-contaminated sensors with spherical interpolation statistically weighted on the basis of all remaining sensors, and (4) computes the variance of the signal across trials to document the stability of the averaged waveform. The rejection of artifact-contaminated trials and sensor epochs relies on the calculation of statistical parameters for the absolute value over time, standard deviation over time, the maximum of the gradient of values over time (first derivative), and the determination of boundaries for each of these three parameters. Data reported here are based on an average reference.

Previously, individual waveform analyses and conventional analysis of variance (ANOVA) were used in concert to demonstrate the selective cortical processing of emotional cues (Schupp et al., 2003a). Individual waveform analyses of each sensor served to identify the temporal and spatial characteristics of the early and late affective ERP modulation. Furthermore, information of this first waveform analysis served to collapse data across time and topography according to the spatial focus and temporal characteristics of the affective modulation, which was submitted to conventional repeated-measures ANOVA. For the purpose of replication, the same analytical plan was used to analyze the present data set. Therefore, individual waveform analyses for each sensor were calculated to determine if the topography and appearance of early and late selective processing of affective pictures were replicated for brief picture presentations. Repeated-measurement ANOVAs including the factor Affect (pleasant vs. neutral vs. unpleasant) and follow-up comparisons were calculated for each time point after picture onset separately for each individual sensor using a criterion of $p<.01$ to identify points of interest. To avoid false positives and to assure a more stringent alpha-level adjustment, these effects were only considered meaningful when the effects were observed for at least eight continuous data points $(32 \mathrm{~ms})$ and two neighboring sensors. These analyses confirmed the presence of early and late selective affective processing, and, importantly, determined significant affective modulation for those sensors identified in the previous study. Therefore, the ANOVAs reported here used sensor clusters identical to those used by Schupp et al. (2003a; see also Figure 1).

Early posterior negativity. The early selective processing was observed in bilateral clusters of enlarged temporo-occipital negativity and corresponding centro-medial positivity for affective compared to neutral cues. Accordingly, early selective affective processing was calculated as area measures in the time interval from 280 to $320 \mathrm{~ms}$ collapsed across left and right temporo-occipital and centro-medial clusters of sensors (as shown in the left panel of Figure 1). This early affective modulation was analyzed with repeated-measures ANOVAs including the factors Affect (pleasant vs. neutral vs. unpleasant), Site (temporooccipital vs. centro-medial), and Laterality (left vs. right).

Late positive potentials. The late selective processing appeared as enlarged late positive potential (LPP) amplitudes for affective relative to neutral pictures over centro-parietal sensors. Accordingly, the late positive potential was scored in the time interval from 400 to $500 \mathrm{~ms}$ averaged over sensors in left and right centro-parietal clusters as shown in the right panel of Figure 1. For statistical analyses, ANOVAs included the factors Affect and Laterality.

For effects involving repeated measures, the Wilks' Lambda procedure was used to correct for violations of sphericity.

Emotional intensity. Further analyses explored the effects of emotional intensity on the appearance of the selective processing of affective cues. Following previous reports (e.g., Schupp et al., 2003a), differences among specific picture contents were assessed within each valence category separately. For pleasant contents, ANOVAs were calculated for the EPN and LPP amplitudes including Picture Content (erotic couples vs. family/babies) and Laterality. Similarly, for unpleasant contents, ANOVAs were calculated for the EPN and LPP amplitudes including Picture Content (mutilations vs. threat) and Laterality. Additional ANOVAs compared each of the four emotional contents with the neutral picture category. 

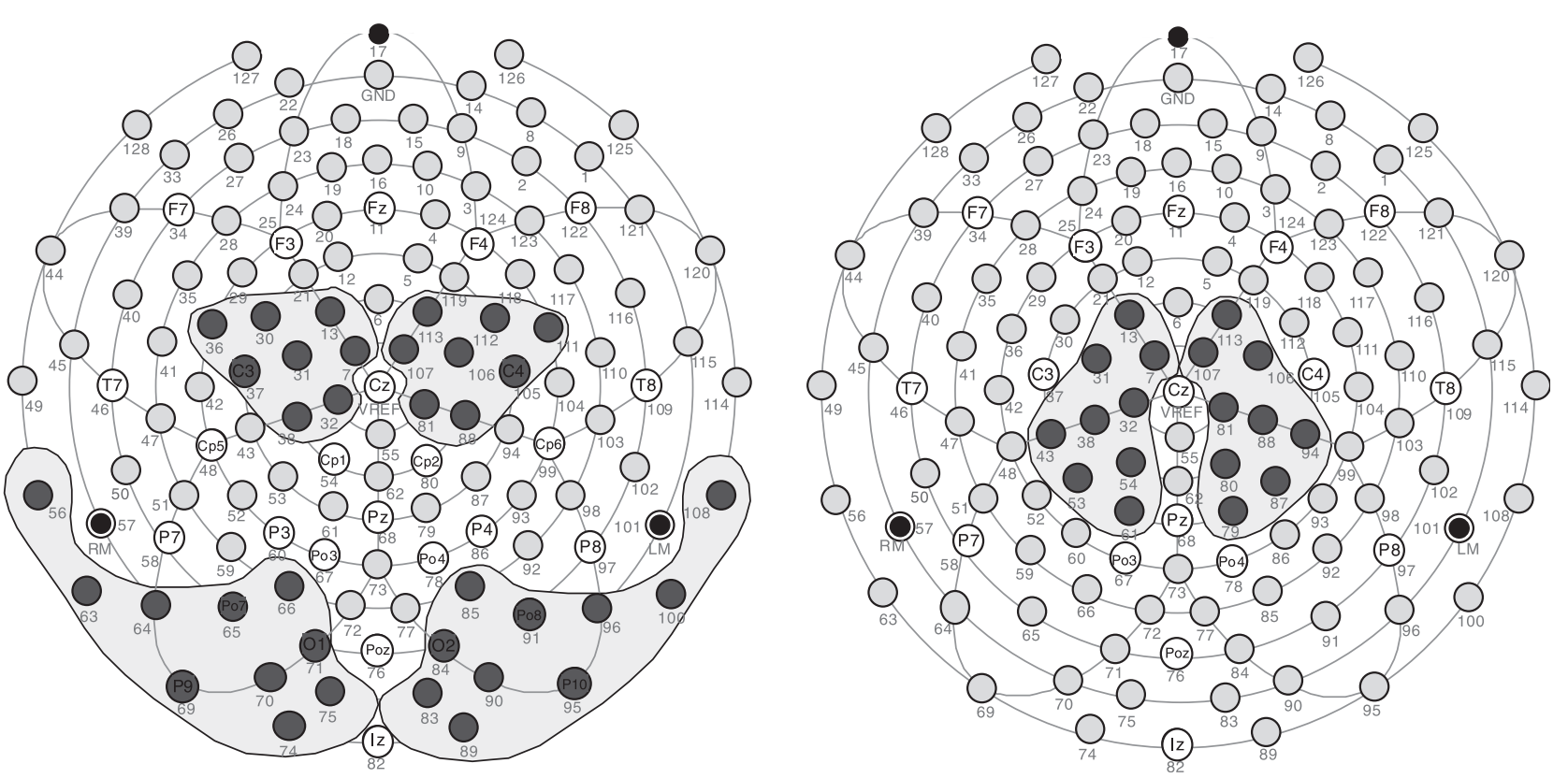

Figure 1. Sensor outline of the geodesic sensor net. The left and right panels illustrate the sensor clusters used to quantify the early (EPN) and late (LPP) selective ERP components, respectively.

\section{Results}

Replicating findings for longer picture presentations (e.g., Schupp et al., 2003a), briefly presented emotional pictures were selectively processed as indicated by increased EPN and LPP amplitudes. The temporal and spatial appearance of the early selective processing of emotional pictures is illustrated in Figures 2 and 3. As shown in Figure 2 for representative central and occipital sensors, the overall topography showed an ERP component developing around $150 \mathrm{~ms}$ and being maximally pronounced around $300 \mathrm{~ms}$, with positive polarity over posterior sensors and negative polarity over centro-medial sites. Superimposed on this waveform, the early emotional modulation appeared as increased negativity over temporo-occipital sites and increased positivity over centro-medial sites. To be consistent with previous research (cf. Junghöfer et al., 2001; Schupp, Öhman, et al., in press), the early selective processing of affective cues is referred to as a relative negative shift (rather than reduced positivity) in the potential waveform over temporo-occipital sites. ${ }^{1}$ Figure 3 illustrates the spatial topography of the affective difference potentials between emotional and neutral images, calculated for the time of strongest affective modulation (280$320 \mathrm{~ms}$ ). Affective images elicited also increased centro-parietal LPP amplitudes compared to neutral images as illustrated in Figure 4. As shown for a left and right parietal individual sensor, increased positivity for affective images developed around 300 $\mathrm{ms}$ and was most pronounced in the time interval from 400 to 600 $\mathrm{ms}$, consistent with previous findings using longer picture

\footnotetext{
${ }^{1}$ Research utilizing the rapid picture presentation paradigm (e.g., Junghöfer, Bradley, Elbert, \& Lang, 2001; Schupp, Junghöfer, Weike, \& Hamm, 2003b) demonstrated a more pronounced negative potential in the same latency range over temporo-occipital sensors. Furthermore, with the notion of a stronger positive potential for neutral compared to emotional materials, some readers might infer stronger neural activation for neutral materials. In contrast, our accompanying fMRI studies indicate increased activation of the extended visual cortex by emotional pictures (Junghöfer et al., 2002).
}

presentations (cf. Schupp et al., 2000). ANOVAs served to confirm the significance of the emotional modulation of the EPN and LPP components.

\section{Early Posterior Negativity}

Affective images were associated with a relative negative shift over temporo-occipital sensors and a relative positive shift over centro-medil sensors, resulting in a significant interaction of Affect $\times$ Site, $F(2,14)=27.0, p<.0001$. As shown below, the calculation of separate ANOVAs for temporo-occipital and centro-medial sensor clusters revealed the expected mirror effects.

Pleasant and unpleasant images elicited significantly larger negativity over temporo-occipital sites compared to neutral images, $\quad F_{\mathrm{S}}(1,14)=65.7$ and 28.7, $p<.0001$; Affect $F(2,14)=39.0, p<.0001$. The calculation of simple difference scores demonstrated that all 16 subjects showed increased negative potentials for pleasant compared to neutral pictures. Similarly, 14 out of 16 subjects showed increased negative potentials for unpleasant relative to neutral images. In addition, the relative negative potential was more pronounced for pleasant than unpleasant pictures, $F(1,14)=24.2, p<.0001$. Although there was no interaction of Affect and Laterality, $F(2,14)=1.5$, n.s., potentials were overall more negative over the right hemisphere, Laterality $F(1,15)=6.3, p<.05$.

The analysis of the centro-medial sensor clusters revealed a significant affective modulation, $F(2,14)=13.7, p<.0001$. Pleasant and unpleasant images were associated with a larger positivity over centro-medial sites compared to neutral images, $F \mathrm{~s}(1,14)=28.3$ and $11.7, p<.001$. In addition, the relative positive shift was more pronounced for pleasant compared to unpleasant pictures, $F(1,14)=4.7, p<.05$. No effects involving Laterality were significant.

\section{Late Positive Potentials}

Replicating previous findings with longer picture presentations, ANOVAs confirmed that the late positive potential was 




right central (\# 107)
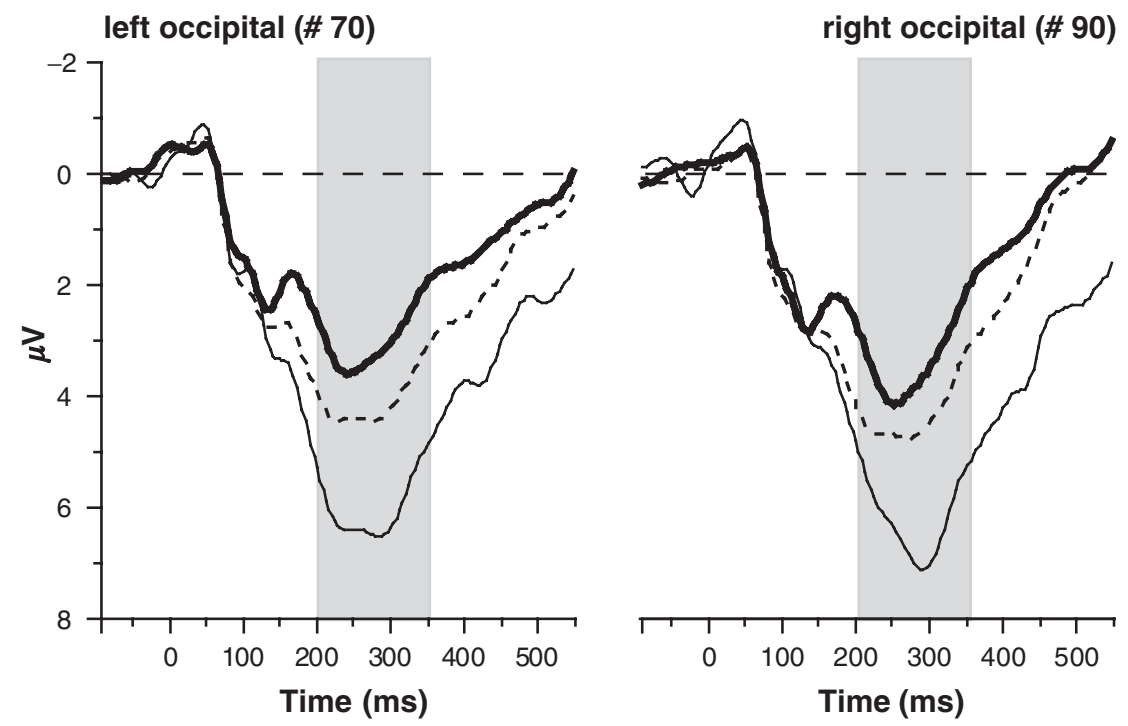

Figure 2. Grand-averaged ERP waveforms of subjects during viewing of pleasant, neutral, and unpleasant pictures. Left and right hemispheric sensors were selected for representing the early posterior negativity (EPN) over the temporo-occipital sites (\#70 and \#90) elicited by pleasant and unpleasant images. The polarity reversal of the affective modulation over centro-medial regions is illustrated by two centro-medial sensors (\#7 and \#107). As highlighted by the gray-shaded area, the EPN was most pronounced in a time window from 200 to $350 \mathrm{~ms}$.

modulated as a function of affect over centro-parietal sensors when pictures were presented briefly, $F(2,14)=30.4, p<.0001$. Pleasant and unpleasant pictures elicited enlarged late positive potentials compared to neutral images, $F_{\mathrm{s}}(1,13)=64.4$ and 46.6, $p<.0001$. Calculating simple difference scores indicated that 15 out of 16 subjects had increased LPP amplitudes to pleasant and unpleasant pictures compared to neutral images. LPP amplitudes to pleasant and unpleasant pictures did not differ, $F_{\mathrm{s}}(1,15)=1.1$, n.s., and no effects involving Laterality were significant, $F_{\mathrm{S}}<1$.

\section{Emotional Intensity}

Further analyses explored the effects of emotional intensity on the appearance of early and late selection of affective cues. Erotic pictures elicit stronger skin conductance responses (SCR) and are rated as more arousing than other pleasant materials (cf. Bradley, Codispoti, Cuthbert, \& Lang, 2001). In the same vein, pictures of mutilated bodies evoke stronger SCRs and are rated as more arousing than other unpleasant contents (cf. Bradley, Codispoti, Cuthbert, et al., 2001; Schupp, Cuthbert, et al., in press). Accordingly, if effects of early and late selection vary as a function of affective engagement, EPN and LPP amplitudes should be most pronounced for contents high in emotional arousal, that is, erotic images and mutilations.

As shown in Table 1, erotic images indeed elicited more pronounced negativity over temporo-occipital sites compared to less arousing pleasant pictures of families and babies, $F(1,15)=14.1, p<.001$, and compared to the neutral category, $F(1,15)=111.0, p<.0001$. In addition, pictures of families and babies were also associated with increased posterior negativity compared to neutral contents, $F(1,15)=27.0, p<.000$. For the unpleasant materials, both contents, mutilations as well as threat, were associated with increased posterior negativity compared to 
Difference Maps: Early Posterior Negativity


Figure 3. Scalp potential maps reveal the topography of the early affective modulation during brief picture presentations. Bilateral foci of temporo-occipital negativity appeared for pleasant and unpleasant pictures. Polarity reversal of the ERP waveform and the affective modulation was observed over centro-medial sites. To derive these brain maps, voltages were interpolated to the scalp surface using spherical splines and back-projected to a model head. Contour lines were spaced every $0.4 \mu \mathrm{V}$. Illustrated is a left- and right-side view of the model head.

the neutral category, $F \mathrm{~s}(1,15)=31.1$ and $20.8, p<.001$. The increased posterior negativity for mutilations compared to threat failed to reach significance.

The analysis of the LPP (lower row in Table 1) revealed that erotic images were associated with enlarged LPP amplitudes compared to less arousing picture categories of the same valence, $F(1,15)=22.0, p<.0001$, and neutral contents, $F(1,15)=108.3$, $p<.0001$. In addition, pleasant pictures of families and babies also showed more pronounced LPP amplitudes relative to neutral contents, $F(1,15)=17.8, p<.0001$. At the aversive site, unpleasant pictures of mutilations elicited larger LPP amplitudes compared to threatening, $F(1,15)=17.7, p<.0001$, and neutral contents, $F(1,15)=46.6, p<.0001$. Pictures of threat also elicited larger LPP amplitudes compared to neutral images, $F(1,15)=38.0, p<.0001$.

\section{Discussion}

Recent studies determined that briefly presented affective pictures engage appetitive and aversive motivational systems as indexed by specific changes in facial EMG activity, startle reflex modulation, and autonomic changes (cf. Codispoti et al., 2001; Globisch et al., 1999). Building upon these findings, the present



right centro-parietal (\# 80)
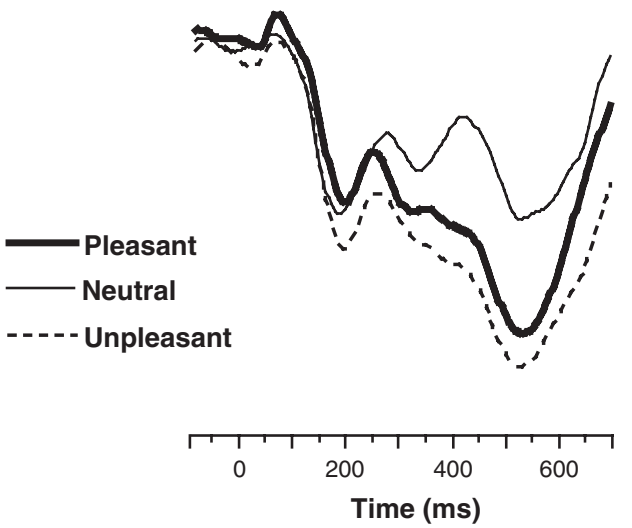

Figure 4. Grand-averaged ERP waveforms over parietal regions to illustrate the late affective modulation. Left and right hemispheric sensors (\#54 and \#80) were selected for representing the enlarged LPP amplitudes elicited by pleasant and unpleasant images. 
Table 1. Peak Amplitude for the Early Posterior Negativity and the Late Positive Potential in Microvolts for Specific Pleasant, Unpleasant, and Neutral Picture Categories Averaged over Left and Right Hemispheric Sites ${ }^{\mathrm{a}}$

\begin{tabular}{|c|c|c|c|c|c|}
\hline \multirow[b]{3}{*}{ ERP component } & \multicolumn{5}{|c|}{ Category } \\
\hline & \multicolumn{2}{|r|}{ Pleasant } & \multirow[b]{2}{*}{ Neutral people } & \multicolumn{2}{|c|}{ Unpleasant } \\
\hline & Erotic images & Family and baby pictures & & Threat pictures & Mutilated bodies \\
\hline EPN & $\begin{array}{c}2.2 \\
(\Delta-3.6)\end{array}$ & $\begin{array}{c}3.5 \\
(\Delta-2.3)\end{array}$ & 5.8 & $\begin{array}{c}3.8 \\
(\Delta-2.0)\end{array}$ & $\begin{array}{c}3.5 \\
(\Delta-2.3)\end{array}$ \\
\hline LPP & $\begin{array}{c}3.5 \\
(\Delta+3.4)\end{array}$ & $\begin{array}{c}1.7 \\
(\Delta+1.6)\end{array}$ & 0.1 & $\begin{array}{c}2.3 \\
(\Delta+2.2)\end{array}$ & $\begin{array}{c}3.2 \\
(\Delta+3.1)\end{array}$ \\
\hline
\end{tabular}

${ }^{a}$ Values in parenthesis for pleasant and unpleasant categories indicate the difference $(\Delta \mu \mathrm{V})$ to the neutral category.

study explored the cortical processing of briefly presented pictures. The results revealed that the selective processing of briefly presented pictures was, in many ways, similar to those found for longer presentations and consistent with the hypothesis that affective pictures draw attentional resources. More specifically, briefly presented affective pictures were associated with early and late selective processing of pleasant and unpleasant pictures (e.g., Schupp et al., 2000, 2003a). The early selective processing of emotional cues was observed as a pronounced relative negative shift in the ERP waveform over temporooccipital leads that started around $150 \mathrm{~ms}$ after picture onset and reached its peak around $300 \mathrm{~ms}$. Late selective processing effects were indicated by enlarged LPP amplitudes to emotional compared to neutral images over centro-parietal sensors. These data suggest that a quick glimpse of emotionally relevant stimuli appears sufficient to tune the brain for the selective processing of emotional pictures.

Functional and evolutionary considerations suggest the benefit of the selective processing of emotional cues, even when briefly presented. In a world where various stimuli compete for attentional resources (cf. Pessoa, Kastner, \& Ungerleider, 2002), the fast and reliable detection of positive and negative reinforcers facilitates adaptive behavior, finally promoting survival and reproductive success (Cacioppo et al., 1999; Lang et al., 1997; Öhman, Flykt, \& Lundqvist, 2000). Accordingly, to reliably detect potentially relevant cues, even a brief glimpse of emotional cues appears sufficient to facilitate perceptual stimulus analysis, and to select affective cues for more elaborate stimulus processing as revealed by augmented EPN and LPP amplitudes. Furthermore, consistent with evolutionary considerations, the selective processing of affective cues varied with the emotional intensity of the pictures. Both early and late selective ERP modulations were most pronounced for pictures prompting highest affective engagement, that is, erotic images and pictures of mutilations (cf. Schupp et al., 2000, 2003a).

Recent data by Junghöfer et al. (2001) corroborate the findings of an early affective discrimination, that is, increased EPN amplitudes to affective stimuli. This research was based on a different experimental approach, the rapid serial visual processing paradigm (RSVP). In this study, 700 IAPS pictures were shown as a continuous stream, while individual pictures were presented for either 333 or $200 \mathrm{~ms}$. Differentiating between emotionally intense and less emotional contents, the results clearly revealed a pronounced posterior negativity elicited by emotionally intense images for both the 333-ms and 200-ms presentations. The main neural sources for the EPN appeared to be located in visual processing areas of the brain. Interestingly, although Junghöfer et al. (2001) employed longer presentation times of individual pictures than the present study, picture processing in the RSVP paradigm was always masked by the upcoming pictures. Thus, the collective finding of these studies is an early selective processing of affective cues as indexed by the EPN, which is observed in widely differing experimental settings.

One concern regarding interpretation of the affective modulation observed during brief and longer picture presentations is that the selective processing of affective cues might depend on some form of evaluative classification of the stimulus materials. Thus, although subjects received no instruction to pay attention to any particular valence category, the effects may be observed only when subjects need to classify the pictures. However, recent studies suggest that this hypothesis is unlikely for either the early or late selective effects. For instance, Junghöfer et al. (2001) observed an early selection of affective cues when subjects simply viewed the pictures and did not perform an explicit categorization task. Moreover, a recent study (Schupp, Junghöfer, Weike, \& Hamm, 2003b) demonstrated increased EPN amplitudes to emotional cues when subjects viewed the pictures and concurrently performed an explicit cognitive attention task that was unrelated to the emotional contents. Furthermore, recent findings indicate that explicit attentional resources can even be directed toward the auditory modality without changing the effects of early selective processing of visual emotional cues (Schupp, Stockburger, et al., 2003). These data suggest that the early selective effects induced by emotional cues do not depend on the emotional categorization of the pictures. In contrast, selective emotional processing appears to reflect a default mechanism, which can be observed while subjects pursue other explicit cognitive goals.

Several studies also observed an affective modulation of the LPP amplitude when subjects did not classify the picture materials. For instance, Cuthbert et al. (1995) compared LPP modulation under conditions when subjects either simply viewed the materials or performed an evaluative SAM rating task and found similar affective modulation in both experimental conditions. Although they did not provide detailed temporal resolution, fMRI studies observed increased BOLD signals in the extended visual cortex while subjects view emotional pictures without being explicitly instructed to classify the pictures (cf. Lang et al., 1998; Bradley et al., 2003; Junghöfer et al., 2002). Although these findings suggest that the selective processing by emotional cues does not depend on the explicit categorization of the pictures, the selective processing of emotional cues might 
depend on the availability of processing resources. For instance, a recent fMRI study, employing a demanding spatial attention task, failed to observe amygdala and fusiform activation when attention was diverted from processing of emotional facial expressions (Pessoa, McKenna, Gutierrez, \& Ungerleider, 2002). Future studies are needed to further probe the competition for selective processing as a function of emotional intensity and explicit attention demands.

However, the present results revealed also some differences for brief picture presentations compared to the more sustained presentations of affective pictures as utilized in previous studies. For instance, briefly presented erotic images elicited a distinctly stronger posterior negativity compared to all other emotional pictures, even highly arousing aversive contents. These observations were not predicted, and, at present, one can only speculate about the meaning of this finding. The present study included primarily female participants, and, thus, gender differences in affective processing need to be considered. Previous studies revealed some gender differences in the processing of pleasant and unpleasant materials. For instance, fMRI data (Lang et al., 1998), PET data (Lane et al., 1997), facial EMG activity, startle reflex modulation, and autonomic measures (Bradley, Codispoti, Sabatinelli, \& Lang, 2001) suggest that women are more responsive to aversive than pleasant contents. However, according to these data, one might expect rather the opposite pattern of modulation for the early selective effects. Thus, further studies are needed to clarify whether the observed differences within the present study are meaningful.

It might be instructive to relate the selective processing of affective cues to the attentional modulation of the ERPs by cognitive events. Both, a pronounced posterior selection negativity and enlarged LPP amplitudes are well-known indices of selective attentional processing in the domain of explicitly directed attention, that is, when subjects are instructed to selectively attend to stimulus features such as color, shape, or orientation (Eimer, 1997; Michie et al., 1999; Näätänen, 1992; Potts \& Tucker, 2001;
Smid, Jakob, \& Heinze, 1999). ${ }^{2}$ Thus, it appears that the intrinsic relevance of affective stimuli and explicit attention are two avenues to induce the selective stimulus processing (cf. Pessoa et al., 2002; Schupp et al., 2003b). Accordingly, albeit more direct evidence awaits future research, these observations are consistent with the hypothesis that affective pictures draw attentional resources. However, the neural structures organizing the selective processing of emotional cues are probably at least partially separate from those involved in explicit selective attention. For example, affective stimuli activate limbic structures such as the amygdala and basal forebrain, which have multiple pathways for limbic regulation of cortical processing (Derryberry \& Tucker, 1991). Indeed, it is proposed that the main difference in network organization between affective and nonaffective representations involves subcortical circuits, allowing for multiple feedforward and feedback projections at various neural levels (Lang et al., 1997; Öhman et al., 2000). Recent data by Anderson and Phelps (2001) suggest that the amygdala might be critical for the enhanced perception of emotional stimuli. Using the attentional blink paradigm, they were able to show that aversive words are better perceived than neutral words and that this enhanced perception of aversive words depended on activation of the amygdala (particularly, the left amygdala).

The processing of motivationally significant stimuli involves adjustments in various neural subsystems ultimately promoting adaptive behavior. It appears that many of the bodily responses can be elicited by brief stimulus exposure, that is, startle reflex, facial, and skin conductance responses (Codispoti et al., 2001; Globisch et al., 1999). In addition, briefly presented emotional pictures are selectively processed as shown by increased EPN and LPP amplitudes. These findings are consistent with the hypothesis that emotional cues prompt a motivational regulation of cortical visual processing and draw attentional resources. A quick glimpse of emotionally relevant stimuli seems sufficient to tune the brain for selective perceptual and postperceptual stimulus encoding.

\section{REFERENCES}

Anderson, A. K., \& Phelps, E. A. (2001). Lesions of the human amygdala impair enhanced perception of emotionally salient events. Nature, 411, 305-308.

Bradley, M. M. (2000). Emotion and motivation. In J. T. Cacioppo, L. G. Tassinary, \& G. G. Berntson (Eds.), Handbook of psychophysiology (pp. 602-642). New York: Cambridge University Press.

Bradley, M. M., Codispoti, M., Cuthbert, B. N., \& Lang, P. J. (2001). Emotion and motivation, I: Defensive and appetitive reactions in picture processing. Emotion, 1, 276-299.

Bradley, M. M., Codispoti, M., Sabatinelli, D., \& Lang, P. J. (2001). Emotion and motivation, II: Sex differences in picture processing. Emotion, 1, 300-319.

Bradley, M. M., Sabatinelli, D., Lang, P. J., Fitzsimmons, J. R., King, W. M., \& Desai, P. (2003). Activation of the visual cortex in motivated attention: Sex and violence. Behavioral Neuroscience, 117, 369-380.

Cacioppo, J. T., Crites, S. L., Jr., Berntson, G. G., \& Coles, M. G. H. (1993). If attitudes affect how stimuli are processed, should they not affect the event-related brain potential? Psychological Science, 4, 108-112.

Cacioppo, J. T., Gardner, W. L., \& Berntson, G. G. (1999). The affect system has parallel and integrative processing components: Form follows function. Journal of Personality and Social Psychology, 76, 839-855.

Christianson, S. A., Loftus, E. F., Hoffman, H., \& Loftus, G. R. (1991). Eye fixations and memory for emotional events. Journal of
Experimental Psychology: Learning, Memory, and Cognition, 17, 693-701.

Codispoti, M., Bradley, M. M., \& Lang, P. J. (2001). Affective reactions to briefly presented pictures. Psychophysiology, 38, 474-478.

Cuthbert, B. N., Schupp, H. T., Bradley, M. M., Birbaumer, N., \& Lang, P. J. (2000). Brain potentials in affective picture processing: Covariation with autonomic arousal and affective report. Biological Psychology, 52, 95-111.

Cuthbert, B. N., Schupp, H., McManis, M., Hillman, C., Bradley, M. M., \& Lang, P. J. (1995). Cortical slow waves: Emotional perception and processing. Psychophysiology, 32, S26.

Derryberry, D., \& Tucker, D. M. (1991). The adaptive base of the neural hierarchy: Elementary motivational controls on network function. In R. A. Dienstbier (Ed.), Nebraska symposium on motivation, 1990: Perspectives on motivation (pp. 289-342). Lincoln, NE: University of Nebraska Press.

Eimer, M. (1997). An event-related potential (ERP) study of transient and sustained visual attention to color and form. Biological Psychology, 44, 143-160.

${ }^{2}$ It is important to note that increased EPN amplitudes to affective cues do not depend on the color of the images. Junghöfer et al. (2001) presented gray-scaled images and observed an almost identical affectmodulated early posterior negativity as for the corresponding color images. 
Globisch, J., Hamm, A. O., Esteves, F., \& Öhman, A. (1999). Fear appears fast: Temporal course of startle reflex potentiation in animal fearful subjects. Psychophysiology, 36, 66-75.

Hamm, A. O., Schupp, H. T., \& Weike, A. I. (2003). Motivational organization of emotions: Autonomic changes, cortical responses, and reflex modulation. In R. J. Davidson, K. Scherer, \& H. H. Goldsmith (Eds.), Handbook of affective sciences (pp. 188-211). Oxford: Oxford University Press.

Junghöfer, M., Bradley, M. M., Elbert, T. R., \& Lang, P. J. (2001). Fleeting images: A new look at early emotion discrimination. Psychophysiology, 38, 175-178.

Junghöfer, M., Elbert, T., Leiderer, P., Berg, P., \& Rockstroh, B. (1997). Mapping EEG potentials on the surface of the brain: A strategy for uncovering cortical sources. Brain Topography, 9, 203-217.

Junghöfer, M., Elbert, T., Tucker, D. M., \& Rockstroh, B. (2000). Statistical control of artifacts in dense array EEG/MEG studies. Psychophysiology, 37, 523-532.

Junghöfer, M., Schupp, H., Stark, R., Schienle, A., Elbert, T., Hamm, A., \& Vaitl, D. (2002). Valence, arousal and selective picture processing: An fMRI analysis. Psychophysiology, 39, S14-15.

Keil, A., Bradley, M. M., Hauk, O., Rockstroh, B. Elbert, T.R, \& Lang, P. J. (2002). Large-scale neural correlates of affective picture viewing. Psychophysiology, 39, 641-649.

Lane, R. D., Reiman, E. M., Bradley, M. M., Lang, P. J., Ahern, G. L., Davidson, R. J., \& Schwartz, G. E. (1997). Neuroanatomical correlates of pleasant and unpleasant emotion. Neuropsychologia, $35,1437-1444$.

Lang, P. J., Bradley, M. M., \& Cuthbert, B. N. (1997). Motivated attention: Affect, activation, and action. In P. J. Lang, R. F. Simons, \& M. Balaban (Eds.), Motivated attention: Affect, activation, and action (pp. 97-135). Mahwah, NJ: Erlbaum.

Lang, P. J., Bradley, M. M., \& Cuthbert, B. N. (1999). International affective picture system (IAPS): Technical manual and affective ratings. Gainesville, FL: The Center for Research in Psychophysiology.

Lang, P. J., Bradley, M. M., Fitzsimmons, J. R., Cuthbert, B. N., Scott, J. D., Moulder, B., \& Nangia, V. (1998). Emotional arousal and activation of the visual cortex: An fMRI analysis. Psychophysiology, 35, 199-210.

Michie, P. T., Karayanidis, F., Smith, G. L., Barrett, N. A., Large, M. M., O'Sullivan, B. T., \& Kavangh, D. J. (1999). An exploration of varieties of visual attention: ERP findings. Cognitive Brain Research, $7,419-450$

Miller, G. A., Gratton, G., \& Yee, C. M. (1988). Generalized implementation of an eye movement correction procedure. Psychophysiology, 25, 241-243.

Näätänen, R. (1992). Attention and brain function. Hillsdale, NJ: Lawrence Erlbaum Asociates.

Öhman, A., Flykt, A., \& Lundqvist, D. (2000). Unconscious emotion: Evolutionary perspectives, psychophysiological data and neuropsycho- logical mechanisms. In R. D. Lane \& L. Nadel (Eds.), Cognitive neuroscience of emotion (pp. 296-327). New York: Oxford University Press.

Pessoa, L., Kastner, S., \& Ungerleider, L. G. (2002). Attentional control of the processing of neutral and emotional stimuli. Cognitive Brain Research, 15, 31-45.

Pessoa, L., McKenna, M., Gutierrez, E., \& Ungerleider, L. G. (2002). Neural processing of emotional faces requires attention. Proceedings of the National Academy of Sciences, USA, 99, 11458-11463.

Potts, G. F., \& Tucker, D. M. (2001). Frontal evaluation and posterior representation in target detection. Cognitive Brain Research, 11, 147156.

Ritter, W., \& Ruchkin, D. S. (1992). A review of event-related potential components discovered in the context of studying P3. In D. Friedman \& G. E. Bruder (Eds.), Psychophysiology and experimental psychopathology: A tribute to Samuel Sutton (pp. 1-32). Annals of the New York Academy of Sciences, Vol. 658. New York: New York Academy of Sciences.

Schupp, H. T., Cuthbert, B. N., Bradley, M. M., Cacioppo, J. T., Ito, T., \& Lang, P. J. (2000). Affective picture processing: The late positive potential is modulated by motivational relevance. Psychophysiology, 37, 257-261.

Schupp, H. T., Cuthbert, B. N., Bradley, M. M., Hillman, C. H., Hamm, A., \& Lang, P. J. (in press). Brain processes in emotional perception: Motivated attention. Cognition and Emotion.

Schupp, H. T., Junghöfer, M., Weike, A. I., \& Hamm, A. O. (2003a). Emotional facilitation of sensory processing in the visual cortex. Psychological Science, 14, 1-7.

Schupp, H. T., Junghöfer, M., Weike, A. I., \& Hamm, A. O. (2003b). Attention and emotion: An ERP analysis of facilitated emotional stimulus processing. NeuroReport, 14, 1107-1110.

Schupp, H. T., Öhman, A., Junghöfer, M., Weike, A. I., Stockburger, J., \& Hamm, A. O. (in press). The selective processing of threat faces: An ERP analysis. Emotion.

Schupp, H. T., Stockburger, J., Bublatzky, F., Junghöfer, M., Weike, A. I., \& Hamm, A. O. (2003). The selective processing of affective pictures: Effects of emotion and attention [Abstract]. Journal of Psychophysiology, 17, 178.

Smid, H. G. O. M., Jakob, A., \& Heinze, H. J. (1999). An event-related brain potential study of visual selective attention to conjunctions of color and shape. Psychophysiology, 36, 264-279.

Tucker, D. M., Liotti, M., Potts, G. F., Russell, G. S., \& Posner, M. I. (1994). Spatiotemporal analysis of brain electrical fields. Human Brain Mapping, 1, 134-152.

(Received September 5, 2002; ACCEPted August 14, 2003) 